\title{
Surgical treatment of non-functioning pancreatic tumor
}

\author{
Alina loana Puscașu ${ }^{*, 1}$, Andra Rădulescu', Răzvan Constantin Anghel ${ }^{1}$, Delia \\ Gabriela Ciobanu Apostol ${ }^{1,3}$, Elena Cotea ${ }^{2}$, Alin Vasilescu ${ }^{1,2}$
}

1 "Grigore T. Popa" University of Medicine and Pharmacy, lasi, Romania ${ }^{2}$ Department of General Surgery, $\left.\right|^{\text {st }}$ Surgical Clinic, lasi, Romania, ${ }^{3}$ Pathology Department, "Sf. Spiridon" Clinical Emergency Hospital, lasi, Romania

\begin{abstract}
Pancreatic neuroendocrine tumors have a rare annual incidence in the general population and they are classified into functional and non-functional tumors. Non-functioning tumors represent the majority. We report an uncommon non-functional neoplasm that developed into the pancreatic isthmus. A 70-year-old woman presented at the hospital with abdominal pain, nausea and vomiting. The abdominal echography revealed a hypoechoic mass of $65 / 54 \mathrm{~mm}$ with dilatation of the upstream Wirsung duct. Non-contrast-enhanced computed tomographic demonstrated a well-enhanced mass of $4 / 5 / 6.5 \mathrm{~cm}$ on the head of the pancreas. An endocrine tumor of the pancreas was considered as a preoprerative diagnosis and pancreatectomy was performed with pancreatojejunum anastomosis- the Beger surgery. As a result, a non-functional endocrine tumor of the pancreas was confirmed anatomo-pathologically. The clinical manifestation of non-functioning endocrine pancreatic tumors is characterized by nonspecific symptoms. They represent a group of neoplasms which can delay the real diagnosis. Currently, CT scans are most used for detection. A rare case of non-functional neuroendocrine pancreatic neoplasm with extensive growth within the head and isthmus part of the pancreas was presented. An early detection of non-functioning endocrine neoplasm of the pancreas is very important and curative resection should be tried for a good prognosis.
\end{abstract}

Keywords: non-functioning pancreatic neuroendocrine tumor, central pancreatectomy, Braun anastomosis, Beger procedure

\section{Introduction}

A neuroendocrine tumor of the pancreas is a rare condition, with an approximated annual incidence of about 5-10 per million in population. Non-functioning neuroendocrine neoplasms of the pancreas are islet cell tumors that are not associated with signs of hormone hypersecretion [1]. Because of the lack of endocrine symptoms, patients usually do not present at the hospital until the symptoms secondary to mass effect manifest, like abdominal pain, weight loss and jaundice.

Received: November 2014; Accepted after review: February 2015; Published: March 2015

*Corresponding author: Alina Ioana Puscasu, "Grigore T. Popa" University of Medicine and Pharmacy, 16 Universitatii str., lasi, Romania.

E-mail: alinaioanapuscasu@yahoo.com
The non-functioning neoplasms of the pancreas are very rare and are difficult in diagnosis. The histopathological differentiation defines the non-functioning pancreatic neuroendocrine tumors. Neuroendocrine cells are characterized by the expression of marker molecules like neuron-specific enolase (NSE), an unspecific cytosolic marker or vesicle proteins like chromogranin $\mathrm{A}$ or synaptophysin, indicating a dense hormonestoring core vesicles and neuropeptides or small neurotransmitter storing synaptic vesicles [2]. The non-functioning neoplasms may have immunohistochemical positivity for neurotransmitters, hormones and neuropeptides. Although most non-functioning tumors show a huge size and histological malignancy with infiltration or invasion to the 
peripheral organs, patients could survive for a long period after tumor resection [3-5]. We present a case of a non-functioning neuroendocrine pancreatic tumor that uniquely grew within the lumen of the Wirsung duct (main pancreatic duct).

\section{Case report}

A 70 year-old woman presented at the hospital with abdominal pain, nausea and vomiting. Laboratory examination revealed increased serum levels of urea $(75 \mathrm{mg} / \mathrm{dl})$, creatinine $(1.59 \mathrm{mg} / \mathrm{dl})$ that could indicate an abnormal renal function. The abdominal echography revealed a hypoechoic mass of $65 / 54 \mathrm{~mm}$ (Figure 1) with dilatation of the upstream Wirsung duct (Figure 2).

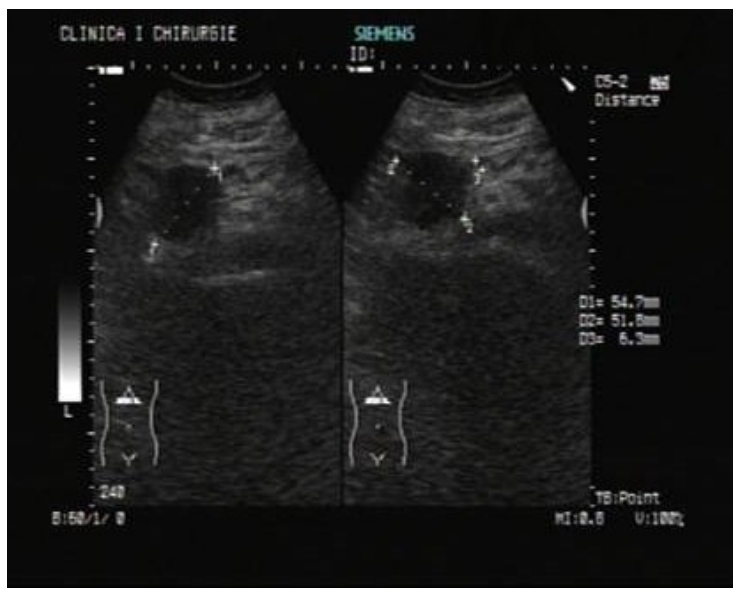

Fig. 1. Sonography: a hypoechoic mass of $65 / 54 \mathrm{~mm}$

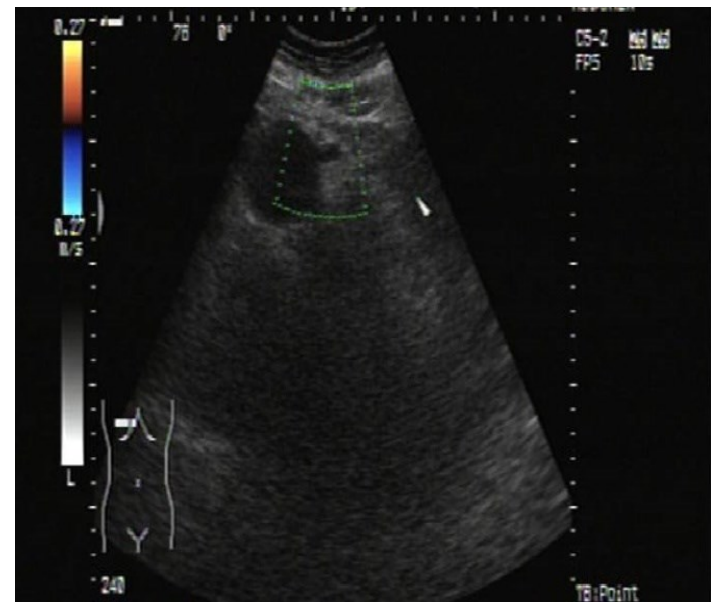

Fig. 2. Sonography: the dilatation of the main pancreatic duct.
Non-contrast-enhanced CT demonstrated a well-enhanced mass of $4 / 5 / 6.5 \mathrm{~cm}$ on the head of the pancreas (Figure 3 ). The mass grew within the lumen of the main pancreatic duct in the head of the pancreas, with upstream dilatation of the Wirsung duct.

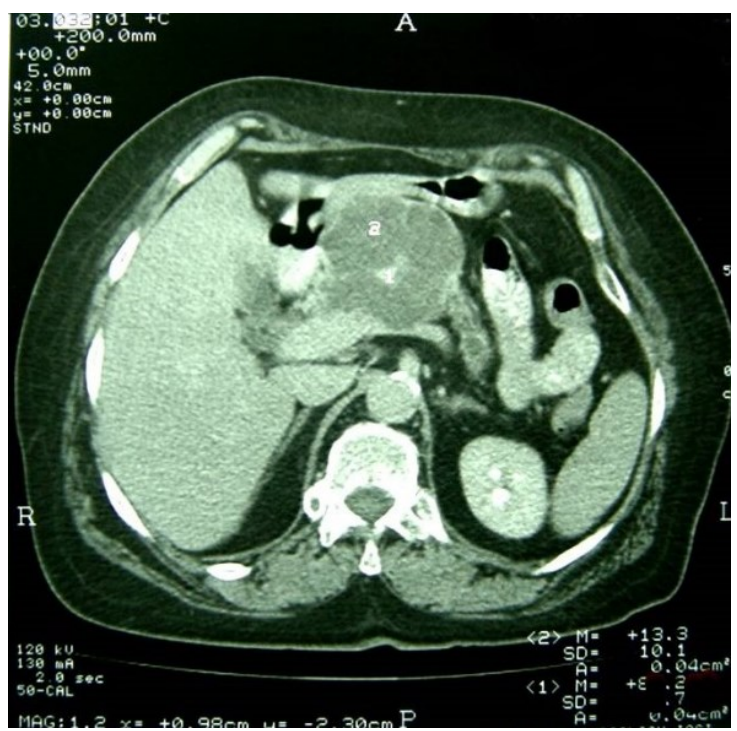

Fig. 3. CT scan: the mass grew in the head of the pancreas, with the dilatation of the Wirsung duct.

Surgical therapy was decided, as the preoperative diagnosis was consistent a neuroendocrine tumor of the pancreas. Under the surgical investigation, we discovered a well-delineated pancreatic tumor mass with diameter of $6 \mathrm{~cm}$, located in the isthmus part of the pancreas, which extended in the head of the pancreas, exteriorized through the small epiplon (Figure 4). The main pancreatic duct (the Wirsung duct) was dilated (Figure 5).

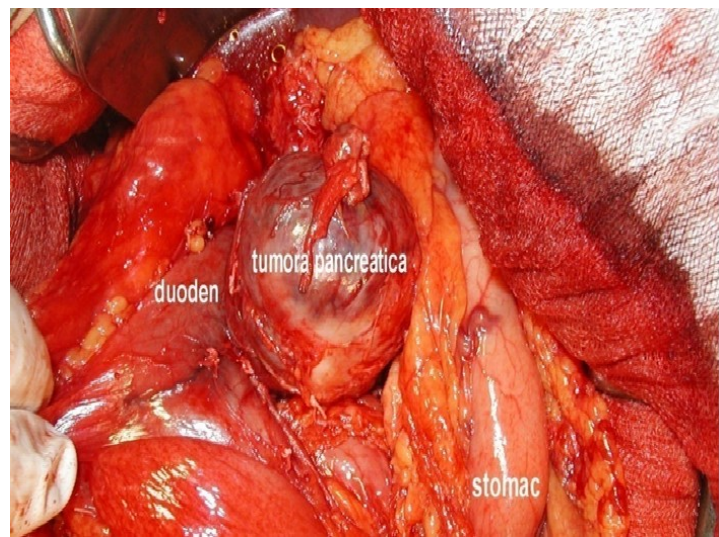

Fig.4. Intraoperatory view: the tumor mass located in the isthmus part of the pancreas, which extended in the head of the pancreas. 


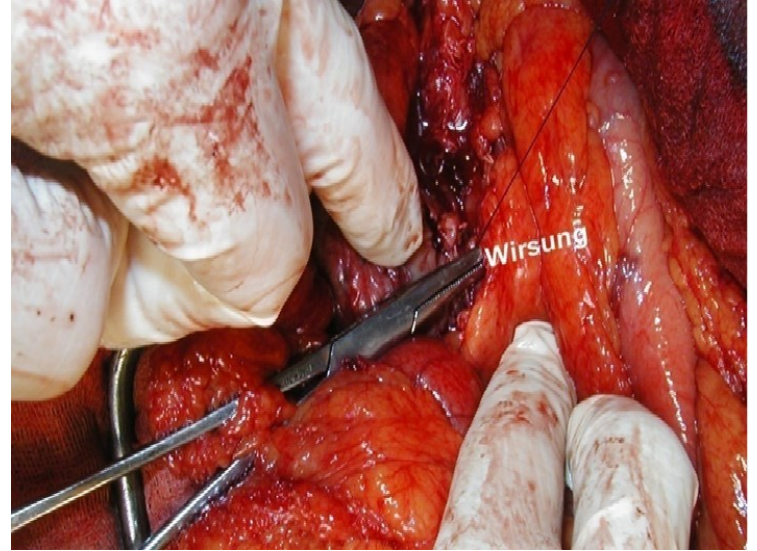

Fig. 5. Intraoperatory view: the dilated main pancreatic duct

The surgical intervention involved a central pancreatectomy with the preservation of the duodenum (Beger procedure) (Figure 6). The digestive tract was restored through a terminolateral pancreatic-jejunal anastomosis (Figure 7).

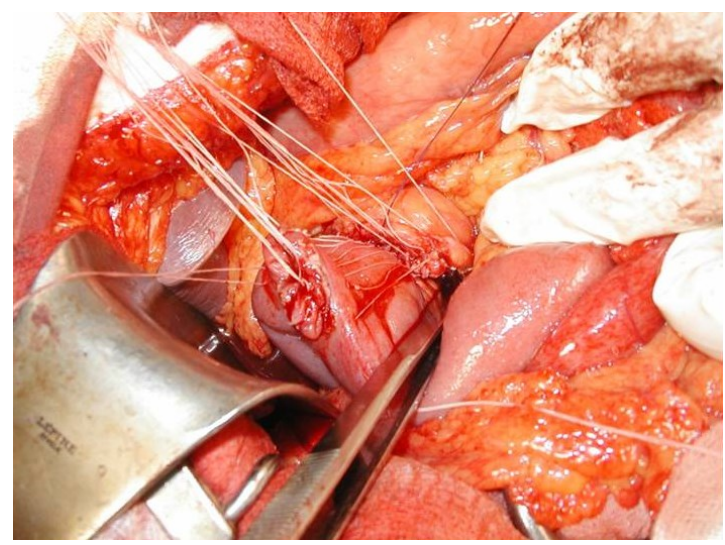

Fig. 6. The termino-lateral pancreatic-jejunal anastomosis

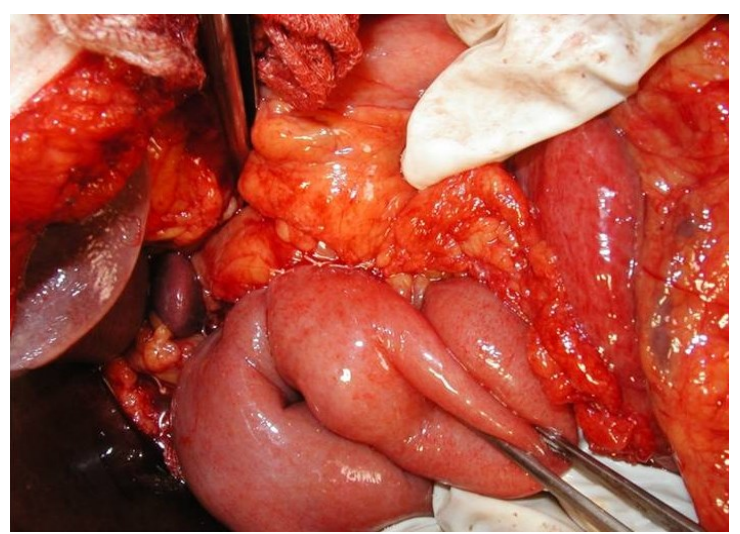

Fig. 7. Braun anastomosis
At gross examination of the pancreatic resection specimen revealed a white tumor mass, apparently encapsulated, with multiple areas of necrosis and hemorrhage (Figures 8 and 9).

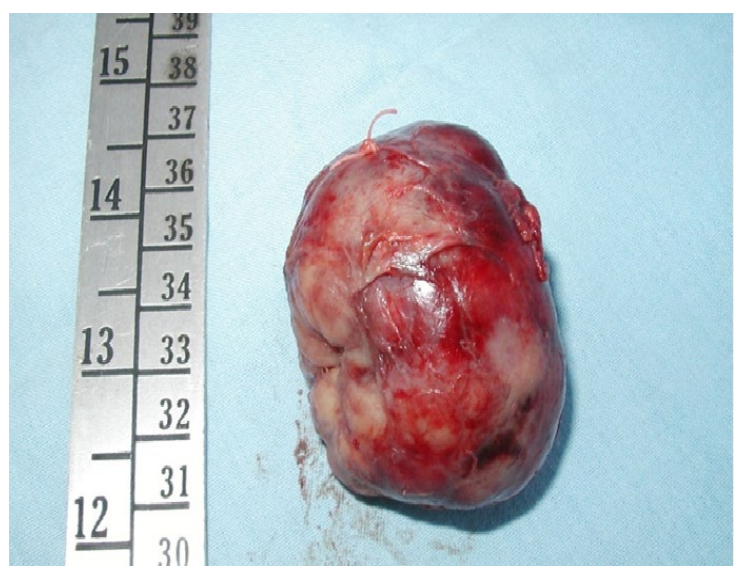

Fig. 8. The pancreatic resection specimen

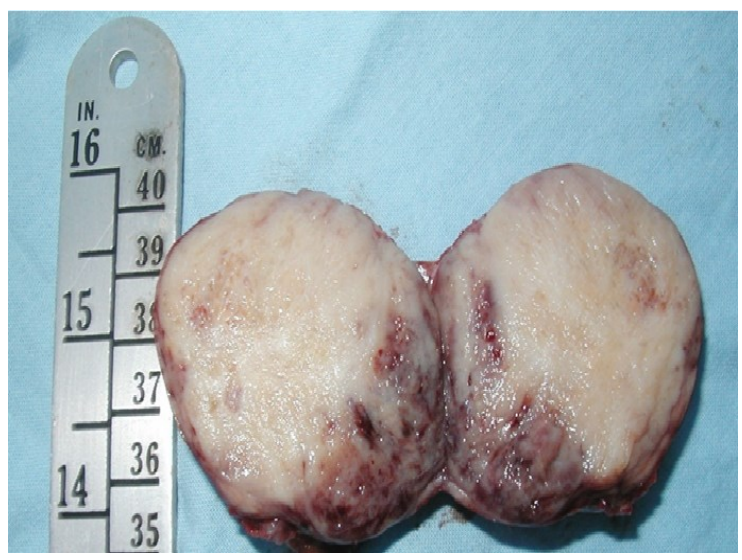

Fig. 9. The tumor mass with multiple areas of necrosis and hemorrhage on cut surface

The surgical resected specimen was fixed in $10 \%$ formalin, embedded in paraffin and microtome-sliced, resulting in $4 \mu$ sections. Subsequently, the sections were routinely stained with hematoxylin and eosin (H\&E staining), while other lesion-specific histochemical staining was also used, namely Van Gieson staining (vG), Congo Red Stain and Grimelius. The immunohistochemical reactions used antibodies that are characteristic for neuroendocrine tumors: Chromogranin, Synaptophysin, NeuronSpecific Enolase (NSE) and CD56. Ki-67 was used to assess the proliferation index while considering the lesion diagnosis and prognosis. The microscopical examination 
showed a solid or tubular architecture with monomorphous cells with round, uniform, centrally located nuclei, in a sclero-hyaline stoma with small, isolated areas of calcification (Figures 10-12).

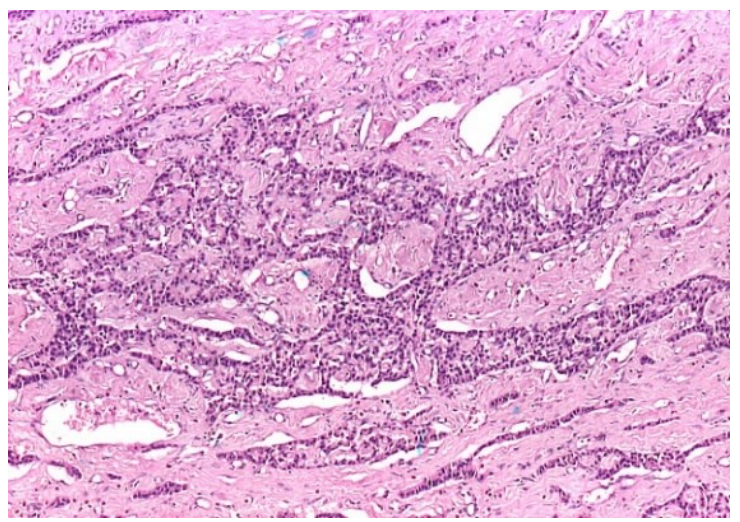

Fig. 10. Solid homogenous structure (HE, x40)

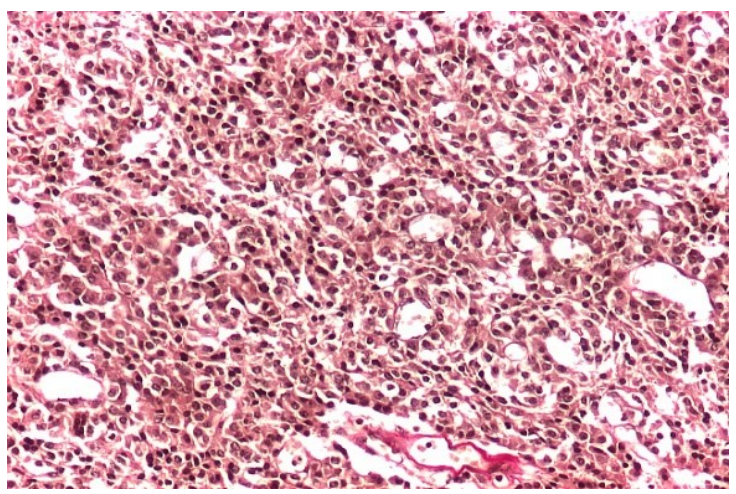

Fig. 11. Tubular and solid architecture (vG, x100)

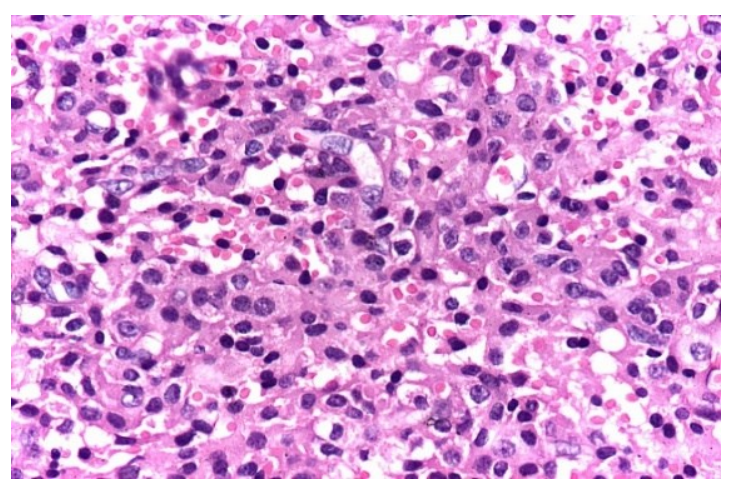

Fig. 12. Monomorphous cells with round, uniform, central nuclei (HE, x200)

The van Gieson staining revealed a collagenous capsule separating the tumor proliferation and the abundant intratumoral sclero-hyaline stroma (Figure 13). Grimelius positivity sustained the endocrine origin of the tumor cells (Figure 14). Van Gieson and
Congo Red reactions excluded the presence of amyloid deposits.

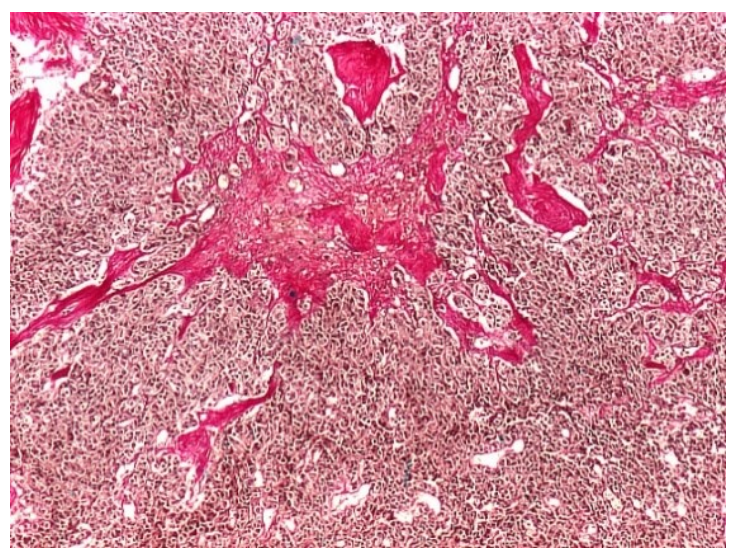

Fig. 13. Sclero-hyaline stroma (vG, x40)

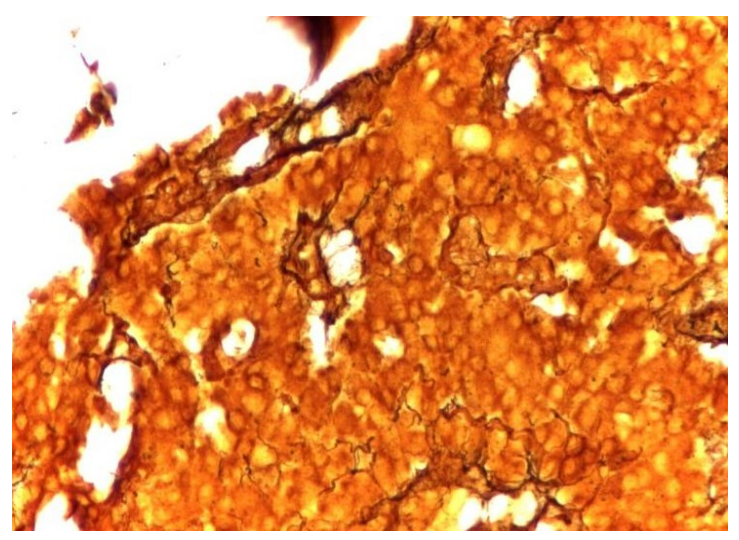

Fig. 14. Positive Grimelius reaction which indicates the endocrine origin of the tumor (Grimelius, x200)

The immunohistochemical reactions confirmed the presence of a neuroendocrine tumor positive for Chromogranin (Figures 15 and 16), Synaptophysin, NSE (Figure 17) and CD56. Ki-67 negativity showed a benign tumorous behavior (Figure 18).

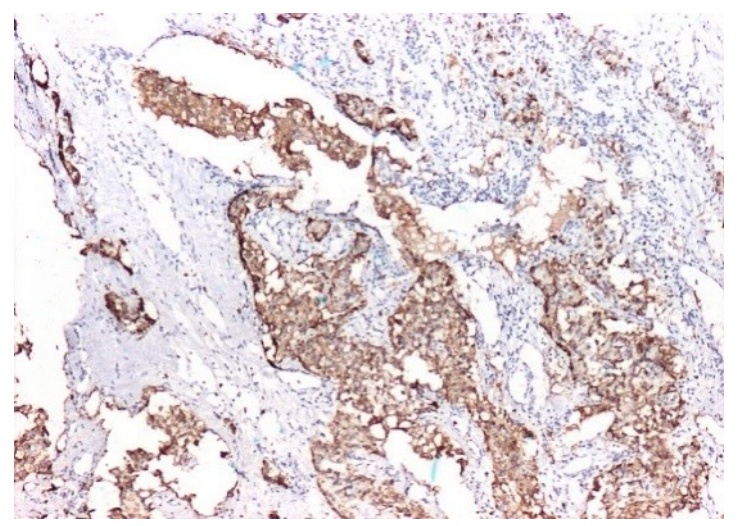

Fig. 15. Intense cytoplasmic positivity of the tumor cells (IHC, Chromogranin, x40) 


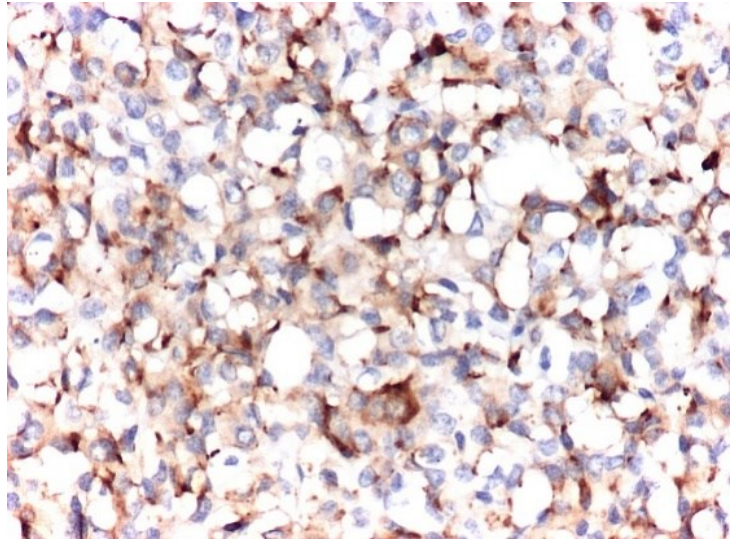

Fig. 16. Cytoplasmic positivity with paranuclear layer strengthening (IHC, Chromogranin, x200)

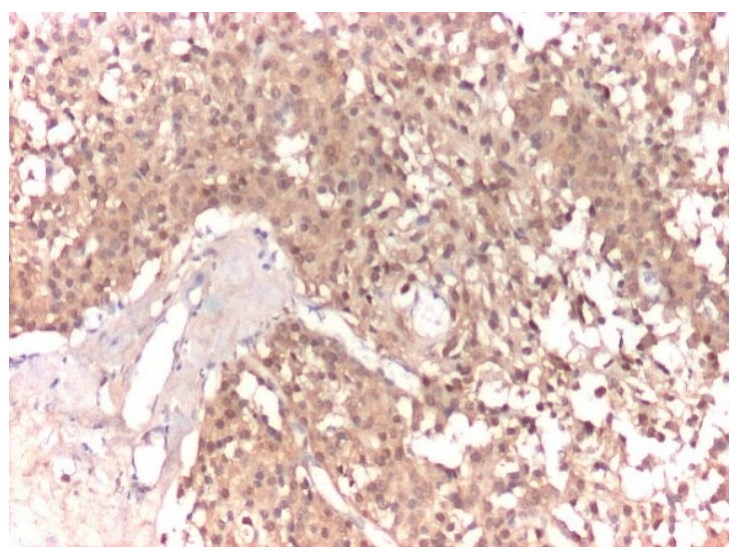

Fig. 17. Positive diffuse marking (IHC, NSE, x100)

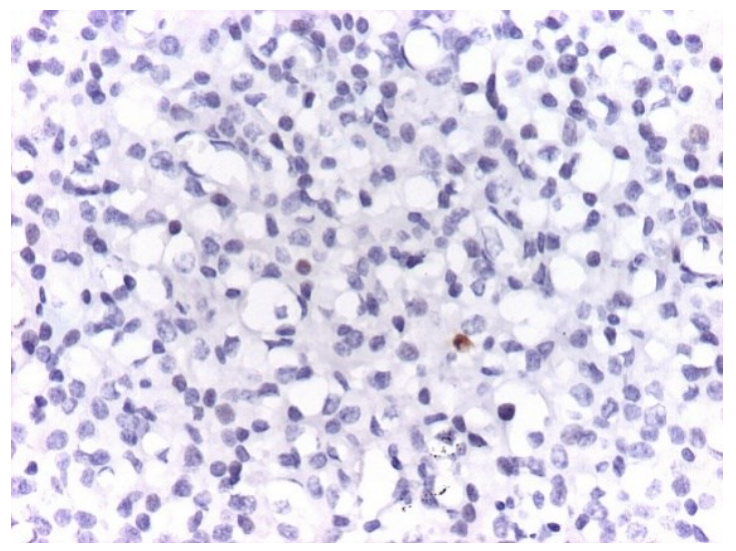

Fig. 18. Ki67 - 0\% (IHC, Ki67, x200)

The pathological examination confirmed the diagnosis of non-functional endocrine tumor of the pancreas.

The postoperative course of the patient was favorable and she was discharged 10 days after surgery. On follow-up at $3,6,12$, and 24 months after the surgical intervention, the patient was in a good general condition.

\section{Discussion}

The clinical manifestation of nonfunctioning endocrine pancreatic tumors is characterized by nonspecific symptoms. They represent a group of neoplasms which can delay the real diagnosis. In most cases, nonfunctioning neuroendocrine pancreatic tumors represent a large abdominal mass that can cause compression of surrounding structures. Imaging techniques such as computer tomography, ultrasound and endoscopic or intraoperative ultrasound have been useful for locating most neuroendocrine pancreatic tumors larger than $2 \mathrm{~cm}$ [6].

Using routine histopathological methods, non-functioning endocrine pancreatic tumors cannot be differentiated from functional tumors. Positive staining with chromogranin A and synaptophysin confirms the diagnosis in both forms $[7,8]$.

In pancreatic endocrine tumors, curative resection improved the survival of many patients with non-functioning neuroendocrine neoplasm of the pancreas. Also, we should have in mind that the surgical procedure differs, depending on tumor characteristics. Small and benign tumors can be removed by enucleation and partial pancreatectomy. In these cases, the pancreas is saved and avoided installing a pancreatic insufficiency. We stress know that if benign neuroendocrine pancreatic tumors are located in the head or isthmus of the pancreas or it is suspected a low malignancy of the tumor, radical resection of the pancreas is indicated to avoid possible tumor recurrence and increase survival rate of the patient [10].

Classic central pancreatectomy involves restoring the pancreatic anastomosis. This goal depends directly on two elements: the density of the pancreatic tissue and the pancreatic-jejuno anastomosis technique. In our case, the surgical technique involved: the loop of the jejunum was included in a omega shape and each limb was separately anastomosed with fragment a pancreatic fragment. In addition, side-to-side jejunojejunostomy of the foot of the omega loop is performed. The peculiarity of the case besides surgical intervention was represented by pathological outcome, which identified the 
tumor as benign although it exceeded $2 \mathrm{~cm}$. This comes in opposition with the WHO 2010 classification criteria that also claim that a neoplasm which has dimensions greater than $2 \mathrm{~cm}$ is considered to have a malignant development [11]. Systemic chemotherapy and radiation therapy have a limited effect on disease progression. Cytotoxic drugs with systemic effect are recommended in patients with liver metastases. Also Peptide Receptor Radionuclide Therapy (PRRT) would provide satisfying results. In patients with localized tumors, radical resection should be considered to obtain a significant prognostic [12].

\section{References}

1. Gullo L, Migliori M, Falconi $M$, et al. Nonfunctioning pancreatic endocrine tumors: A multicenter clinical study. Am J Gastroenterol. 2003; 98(11):2435-2439.

2. Falconi M, Plockinger U, Kwekkeboom DJ, et al. Well-differentiated pancreatic nonfunctioning tumors/carcinoma. Neuroendocrinology. 2006; 84(3):196-211.

3. Farkas G. Surgical treatment of neuroendocrine tumors of the pancreas. Magy Onkol. 2003; 47(4):345-347.

4. Sarmiento JM, Que FG, Grant CS, Thompson GB, Farnell MB, Nagorney DM. Concurrent resection of pancreatic islet cell cancers with synchronous hepatic metastases: outcomes of an aggressive approach. Surgery 2002; 132(6):976-982.

5. Matthew BD, Smith TI, Kercher KW, Holder WD Jr, Heniford BT. Surgical experience with functioning pancreatic neuroendocrine tumors. Am Surg. 2002; 68(8):660-5.

6. Kazuhiro $\mathrm{H}$. Recent standardization of treatment strategy for pancreatic neuroendocrine tumors. World J Gastroenterol 2010; 16(36):4519-4525.

7. Appetecchia M, Baldelli R. Somatostatin analogues in the treatment of gastro-

\section{Conclusion}

We presented a rare case of nonfunctioning neuroendocrine pancreatic neoplasm with extensive growth within the head and isthmus part of the pancreas was presented. An early detection of nonfunctioning endocrine neoplasm of the pancreas is very important and curative resection should be tried for a good prognosis.

enteropancreatic neuroendocrine tumours, current aspects and new perspectives. J Exp Clin Cancer Res 2010; 29:19.

8. Haynes $A B$, Deshpande $V$, Ingkakul $T$, et al. Implications of incidentally discovered, nonfunctioning pancreatic endocrine tumors: short-term and long-term patient outcomes. Arch Surg. 2011; 146(5):534-538.

9. Balzano $G$, Zerbi A, Veronesi $P$, Cristallo M, Di Carlo V. Surgical treatment of benign and borderline neoplasms of the pancreatic body. Dig Surg. 2003; 20(6):506-510.

10. Yamaguchi K, Yokohata K, Ohkido $\mathrm{M}$, et al. Which is less invasive - distal pancreatectomy or segmental resection. Int Surg. 2000; 85(4):297-302.

11. Triponez F, Goudet $P$, Dosseh, et al. Is surgery beneficial for MEN-1 patients with small (< or = $2 \mathrm{~cm})$, nonfunctioning pancreaticoduodenal endocrine tumor? An analisys of 65 patients from the GTE. Word J Surg 2006; 30(5): 654662.

12. Kang CM, Kim KS, Choi JS, Lee WJ, Kim BR. Experience with nonfunctioning neuroendocrine neoplasms of the pancreas. Dig Surg 2005; 22(6):453-458. 